\title{
A randomised trial of continuous skin-to-skin contact after preterm birth and the effects on salivary cortisol, parental stress, depression, and breastfeeding
}

Eva-lotta Mörelius, Annika Örtenstrand, Elvar Theodorsson and Anneli Frostell

\author{
Linköping University Post Print
}

\section{Tweet}

N.B.: When citing this work, cite the original article.

Original Publication:

Eva-lotta Mörelius, Annika Örtenstrand, Elvar Theodorsson and Anneli Frostell, A randomised trial of continuous skin-to-skin contact after preterm birth and the effects on salivary cortisol, parental stress, depression, and breastfeeding, 2015, Early Human Development, (91), 1, 6370.

http://dx.doi.org/10.1016/j.earlhumdev.2014.12.005

Copyright: Elsevier http://www.elsevier.com/

Postprint available at: Linköping University Electronic Press http://urn.kb.se/resolve?urn=urn:nbn:se:liu:diva-113796 
A randomised trial of continuous skin-to-skin contact after preterm birth and the effects on salivary cortisol, parental stress, depression, and breastfeeding

\section{Abstract}

Aim: To evaluate the effects of almost continuous skin-to-skin contact (SSC) on salivary cortisol, parental stress, parental depression, and breastfeeding.

Study design: A randomised study engaging families of late preterm infants (32-35 weeks gestation). Salivary cortisol reactivity was measured in infants during a nappy change at one month corrected age, and in infants and mothers during still-face at four months corrected age. Both parents completed the Swedish Parenthood Stress Questionnaire (SPSQ) at one month and the Edinburgh Postnatal Depression Scale (EPDS) at one and four months. Ainsworth's sensitivity scale was used to control for parental sensitivity.

Subjects: Thirty-seven families from two different neonatal care units in Sweden, randomised to either almost continuous SSC or standard care (SC).

Results: Infants randomised to SSC had a lower salivary cortisol reactivity at one month $(\mathrm{p}=0.01)$. There was a correlation between the mothers' and the preterm infants' salivary cortisol levels at four months in the SSC group $(\rho=0.65, p=0.005)$, but not in the SC group $(\rho=0.14, p=0.63)$. Fathers in SSC scored lower on the SPSQ sub-scale spouse relationship problems compared to fathers in $\mathrm{SC}(\mathrm{p}<0.05)$.

Conclusions: Almost continuous SSC decreases infants' cortisol reactivity in response to handling, improves the concordance between mothers' and infants' salivary cortisol levels, and decreases fathers' experiences of spouse relationship problems. 


\section{Introduction}

Preterm infants in neonatal intensive care are treated in a stressful environment and are exposed to several stressful interventions every day [1]. On top of that, the preterm infant is commonly separated from the mother. Mothers separated from their new-born infants often describe the separation as a major stressor [2]. Infants separated from their mother express a distress call that stops when the infant is returned to the mother [3]. Animal studies show that rodent pups are more sensitive to stress if they have been separated from their dams [4]. The combination of exposure to a stressful environment, stressful procedures, maternal separation and brain immaturity increases the risk of disturbances in the hypothalamus-pituitary-adrenal (HPA) axis [5-7].

The HPA axis regulates cortisol production and the organism's capacity to respond to stressors. The HPA axis organises in early infancy in response to the environment, for example maternal contact or deprivation [4]. Maternal contact can buffer infant stress, while deprivation leads to higher sensitivity to stress [8]. Studies of rodents have shown that maternal licking and grooming alter the glucocorticoid receptor gene expression in the brain, lowering the corticosterone (rodents' equivalent to cortisol) reactivity [9]. On the other hand, social isolation, increases the corticosterone reactivity in mice [10]. It has been suggested that environmental factors, such as spending time together and sharing the same environment, can enhance a concordance between the mother's and the infant's cortisol levels [11-16]. A correlation between mothers' and preterm infants' cortisol levels at discharge from the NICU has been described when the mothers were staying together with their infants, sharing the same environment around the clock [17]. Such a correlation in salivary cortisol levels was not found when the mother was not allowed to stay overnight at the ward [17]. A correlation in cortisol levels between twins sharing the same environment has also been described [17-19]. 
Parents need to be present and stay close to their infant to have the possibility to cherish and support the infant and thus buffer stress reactions. Therefore, providing facilities for parents to stay in the NICU to enable sharing the infant's environment is an important intervention [20]. Kangaroo Mother Care (KMC) is an intervention that has been used in neonatal care around the world for many years. The main components of KMC includes skin-to-skin contact (SSC), where the infant lies between the parent's breasts firmly attached to the chest in an upright position, frequent and exclusive or near-exclusive breastfeeding, and early discharge from hospital [21]. SSC have proved to be beneficial for preterm infants, e.g. it contributes to cardiorespiratory stabilisation, fewer signs of stress, and a more organised sleep-wake cycle [22-24]. SSC may also improve co-regulation of salivary cortisol between mother and infant, attenuate the stress reactivity, and buffer the preterm infant's pain reaction during painful procedures [25-27]. For mothers, SSC reduces stress and postpartum depression, improves mood, encourages her to create a stimulating and caregiving environment, and to breastfeed [24, 28-31]. In Sweden, SSC is initiated for extremely preterm infants at a median postnatal age of six days [32]. During the first days, the extremely preterm infant usually experiences SSC for a few hours a day. However, along with maturation, more and more hours may be spent in SSC with the parents, and eventually parents may carry their preterm infant in almost continuous SSC (i.e. almost around the clock) [33].

The experience of having an infant hospitalised in the neonatal intensive care unit is a highly stressful event for parents. Parents of preterm infants experience high stress associated with anxiety, uncertainty and parental role alteration [2, 34, 35]. Gray et al compared stress in mothers of preterm infants with mothers of full-term infants [36]. They found significantly higher stress scores (measured with the parenting stress index) in mothers of preterm infants when the child was one year [36]. The various emotional responses including stress-related symptoms and depression experienced during the infant's first year may affect parents' ability 
to handle their parenthood and thus affect the development of the child. A number of studies indicate that postnatal depression can, besides being a severe state for the parent, be negative for the infant's development because it hinders the parent from detecting, interpreting and responding to the infant's signals in a sensitive and predictable way [37-39] Depressed parents tend to be more passive and non-engaged in the interaction with the infant [40]. They also display more irritability and hostility towards the infant [40]. Infants to parents with depression are less good at self-regulating their emotions compared with infants to parents without depression [39]. However, there are some studies indicating that SSC can lower the risk for postnatal depression and parenting stress [24, 28, 37].

Recently, Neu et al presented data from a trial where mothers were holding their preterm infants for one hour daily for eight weeks in either SSC, in a blanket or free of choice (control group). They found decreased cortisol levels in both mothers and infants in all three groups but no effect on cortisol co-regulation [41]. Neu et al concluded that stress probably needs to be present in order for mothers and infants to demonstrate co-regulation of cortisol levels [41]. The most stress provoking intervention for infants besides separation from the parent is painful procedures [42]. However, to perform painful procedures without pain relief is not considered ethical. A nappy change has previously been used to evaluate stress reactivity in infants [43]. A nappy change is classified as a handling stressor for infants under three months age [42]. Still-face is a well used method to study infants' reactions to contradictory messages from the mother [44]. Still-face is best studied before six months age and is classified as a psychological stressor that elicits negative emotions [42].

Our thought is that if preterm infants are allowed to experience SSC around the clock, they may feel safe in a parent's care, receive the familiar olfactory stimuli and warmth from the 
parent's skin, and hear their heartbeat. All of these factors together may facilitate the maturation of the HPA axis and buffer the infant's stress reactivity. If parents are allowed to stay in the NICU and experience SSC day and night, they may be more tuned in to the infant's needs because they can feel all the infant's movements, and may be present the few minutes the infant is awake. The constant closeness may also influence the regularity of a day-night rhythm between the parent and infant, and regulate the cortisol levels accordingly. However, a supportive maternal caregiving behaviour, such as high sensitivity and responsiveness to the infant's signals [45], may also buffer the infant's response to stressors, resulting in a lower rise in cortisol [46-50]. Hence, it is important to control for such quality.

Aim

The aim of the present study was to evaluate the effect of almost continuous SSC for late preterm infants (32-36 weeks gestation). Primary outcome was salivary cortisol reactivity in response to two different stressful but non-painful situations at one and four months corrected age. Secondary outcome was to study the concordance between the mothers' and the preterm infants' salivary cortisol levels at four months corrected age. A third outcome was to study parental stress, depression, and breastfeeding.

\section{Methods}

Design

A randomised trial comparing outcomes of almost continuous SSC in one level-III NICU at Linköping University Hospital and one level-II NICU at Södersjukhuset, Sachs’ Children's Hospital in Stockholm. Recruitment to the study took place between April 2008 and April 2012. The infants were randomly allocated to almost continuous SSC or standard care (SC) in relation to delivery by a staff nurse (Figure 1). Allocation was done by sealed, opaque 
envelopes. The study was approved by the Regional Ethical Review Board at Linköping University (M30-07).

\section{Setting}

The health care system in Sweden allows both parents to stay in the NICU, and more and more NICUs offer family rooms [17, 20]. Both NICUs participating in the present study are classified as family-centred wards, and they have 17 and 13 infant beds, respectively. There are separate rooms for each family, including beds for the infant and both parents, a private bathroom, and equipment for supplementary oxygen and telemetric wireless monitoring.

\section{Definitions}

In the current study, SSC was defined as almost continuous SSC, beginning in the delivery room and continuing almost 24 hours a day with the parents alternating until hospital discharge. In the current study, SC means that both parents had the opportunity to practice as much skin-to-skin contact as they liked.

Inclusion criteria

Healthy women about to give birth vaginally to a single preterm infant (GA $32^{0}-35^{6}$ ) without known malformation were eligible for participation. Participating mothers had to be able to read and speak Swedish.

Procedure

\section{In the hospital}

Parents were provided with written and oral information about the study when arriving in the hospital, i.e. when labour had started. Parents who agreed to participate were randomly assigned to either almost continuous SSC or SC. If parents were assigned to almost 
continuous SSC, the infant was placed in an upright position on the parent's chest immediately after birth. The dyad was transferred to the NICU in a SSC position. Parents were provided with clothes, i.e. tubetops, scarves, and blouses to facilitate the care. If the family was assigned to SC the mother and the infant were separated from each other in the maternity ward and transferred to the NICU. Infants in both groups were equipped with a wireless cardio-vascular monitor to control for bradycardias and apnoeas. Axillar body temperature was recorded twice a day, weight once a day and bradycardias and apnoeas when/if occurring. Parents in SSC were told to note who provided the skin-to-skin contact, and if and how long they were off skin-to-skin contact for any reason, while parents in SC were told to note when and how long they provided skin-to-skin contact. Parents have previously shown to be reliable recorders [51]. All parents in both groups were given one lesson in how to notice and respond to a preterm infant's signals. This structured lesson was provided by a NIDCAP ${ }^{\circledR}$ certified nurse. Except for this lesson, the infants and their parents received the same care as everybody else in the NICU.

At the time of discharge from the ward, parents completed a questionnaire of descriptive data and the infant's medical data was collected from the journal. In addition, SSC was not recommended during the night at home, in accordance with recommendations to avoid cosleeping so as to lower the risk of sudden infant death syndrome [52].

\section{One month corrected age}

A home-visit was carried out when the infant was one month corrected age. This visit included data collection consisting of saliva sampling on infants before and after a videotaped nappy change performed by the mother, questionnaires, and an interview with the mother (not presented in this paper). 
A second home-visit was carried out when the infant was four months corrected age. This visit included data collection through questionnaires and saliva sampling on mother and infant before and after a video-taped face-to-face interaction session between mother and infant (still-face [44]), the results of the procedure are not presented in this paper]. The session consisted of 2 minutes interaction, 2 minutes still-face, and 2 minutes interaction.

\section{Measurements}

\section{Salivary cortisol}

Saliva was collected before (baseline) and 30 minutes after (response) a nappy change performed by the mother [53] at one month and during a face-to-face interaction session (still-face) [54] at four months, respectively. Saliva was collected from mothers and infants using cotton buds, as described previously $[55,56]$. Due to components that can disturb the salivary cortisol analysis, saliva was collected at least one hour after food intake. After collection, the saliva was centrifuged, frozen at $-20^{\circ} \mathrm{C}$, and stored at $-70^{\circ} \mathrm{C}$. A radioimmunoassay for cortisol was used to analyse cortisol concentrations in the saliva (Orion Diagnostica, Turku, Finland) [55]. Samples were run in duplicate, and all samples from each individual were run in the same assay. Intra-assay coefficients of variation were $4.6 \%$ and $9.2 \%$ for low and high, respectively.

\section{The Swedish Parenthood Stress Questionnaire}

The Swedish Parenthood Stress Questionnaire (SPSQ) is a Swedish version of the Parenting Stress Index $[57,58]$. It is a self-administrated questionnaire designed to study parental experiences of stress in the parent-child system. The SPSQ consists of 34 items scored on five-point Likert-type scales, where higher scores indicate more parenting stress [58]. Parents are asked to mark the degree to which they agree or disagree with each statement. The items 
are divided in five sub-scales: incompetence, role restriction, social isolation, health problems, and spouse relationship problems. The SPSQ, previously validated in a Swedish population [58], was completed by both parents, separately, at one and four months.

\section{Edinburgh Postnatal Depression Scale}

The Edinburgh Postnatal Depression Scale (EPDS) is a self-administrated questionnaire designed to discover depressions in new mothers [59]. The EPDS consists of 10 items scored from zero to three. Parents are asked to mark which of the given statements that agrees most with their mood in the last seven days. The EPDS, previously validated in a Swedish population with a cut-off point of 11/12 [60], was completed by both parents, separately, at the four-month follow-up. For men, the EPDS has been validated in Australia with an optimum cut-off point of 9/10 [61].

\section{Questions of health and breastfeeding}

A questionnaire was constructed including questions about infants' health, weight, and feeding status. The questionnaire was completed at the follow-ups at one and four months. Mothers were also asked the following questions about breastfeeding: During the pregnancy, did you think about breastfeeding? If yes, was it your wish to breastfeed after delivery?

\section{Ainsworth's sensitivity scale}

To monitor for parental sensitivity, mothers' sensitivity towards their infants' signals was measured with Ainsworth's sensitivity scale (scale 1, sensitivity versus insensitivity) [45]. Ainsworth's sensitivity scale is a nine-point bi-dimensional scale ranging from 1=highly insensitive to 9=highly sensitive. Mothers' sensitivity was rated from video-tapes of the nappy change at one month corrected age. All ratings were made by the same highly 
experienced neonatal nurse (Lisbet de Jounge), who was blinded to randomisation and did not participate in the home-visits.

\section{Statistics}

Non-parametric statistics was used to calculate potential differences between groups (i.e. demographic and medical information, raw baseline cortisol levels, SPSQ, and EPDS), and within groups (e.g. differences between one and four months).

General linear models (GLMs) were used to investigate possible factors influencing infants' and mothers' cortisol reactivity. Cortisol reactivity was calculated as absolute $\Delta$ value. All subjects were their own controls. Because the salivary cortisol values were not normally distributed the cortisol reactivity levels were calculated from log transformed values. Cortisol reactivity was used as dependent variable while randomization group and maternal sensitivity (the Ainsworth's scale) were used as fixed factors in the GLMs. Because the study was made in two hospitals, hospital site was used as a covariate. Concordance between raw cortisol levels in mothers and infants was calculated with Spearman rank-order correlation coefficient. Sample size was based on previous results [24]. Data was analysed using the statistical software SPSS 20.0. Statistical significance was considered if $\mathrm{p}<0.05$.

\section{Participants}

Forty-two families agreed to participate; 23 were randomised to almost continuous SSC and 19 to SC (Figure 1). Because the families were randomised prior to the infants' birth, three families were excluded after birth (born full-term $n=1$, mother unhealthy $n=1$, and delivered with caesarean section $n=1$ ). These families were all randomised to almost continuous SSC. Two families from the SSC group withdrew from the study after two and three days, respectively (Figure 1). In one of the families the mother had diabetes and the father described 
the situation as very stressful because the mother had to stay at the maternity ward for care. Their infant was born in week 35 and the father found it hard to carry the infant so much, and hard to sleep. The other family withdrew without any further explanation after their infant was born in week 33. There were no significant differences in infants' birth weight, age at birth, or gender or parental demographics between the two groups (Table 1).

No infant received steroids during the hospital stay, two infants in the SSC group were administered with antibiotics due to suspected infection. There were no significant differences in the number of bradycardias and apnoeas or in body temperature between the groups during the first five days in the hospital (data not shown). There was no significant difference in age and weight between groups at discharge (Table 1).

\section{Results}

Even though the parents in the SC group were allowed to practice as much skin-to-skin care as they wished, there were significant differences in the number of hours of skin-to-skin contact between the groups during day 2-9 (Table 2). Infants in SSC experienced an average of 19.6 hours of skin-to-skin contact per day during the first week of life, compared to infants in SC who experienced an average of 7.0 hours per day (mostly during daytime).

There were no significant differences in the infants' weight at one or four months corrected age (Table 1). At one month, five parents in each group reported that the infant had had an infection (cold or eye-infection) since discharge. At four months, eight and nine parents in SSC and SC respectively reported that the infant had had an infection (cold, eye-infection, or fever) since the one-month visit. 


\section{Salivary cortisol}

One hundred percent of the collected saliva samples at one and four months were eligible for analysis. There was no significant difference in baseline cortisol levels between SSC and SC at any time point, neither for infants nor mothers (Table 3). The median baseline salivary cortisol level in the SC group was significantly higher at four months compared to one month $(\mathrm{p}=0.04)$ (Table 3).

Using a GLM and statistically controlling for mothers' sensitivity and hospital site revealed a significantly smaller salivary cortisol reactivity for infants in the SSC group compared to infants in the SC group at one month $\left(\mathrm{F}_{(1)}=7.6, \mathrm{p}=0.01\right)$ (Figure 2), but not at four months $\left(F_{(1)}=0.6, p=0.45\right)$. There was no significant difference in mothers' salivary cortisol reactivity at four months between SSC and $\mathrm{SC}\left(\mathrm{F}_{(1)}=0.3, \mathrm{p}=0.58\right)$.

There was a significant correlation between the mothers' and the preterm infants' raw baseline salivary cortisol levels at four months corrected age in the SSC group $(\rho=0.65$, $\mathrm{p}=0.005)$, but not in the SC group $(\rho=0.14, \mathrm{p}=0.63)$.

\section{Parental stress}

There were no significant differences in total SPSQ, or in the sub-scales incompetence, role restriction, social isolation, and health problems, between SSC and SC at one or four months (Table 4). At four months, fathers in SSC scored significantly lower on the sub-scale spouse relationship problems compared to fathers in SC $(\mathrm{p}<0.05)$. Medians (q1-q3) were $2.0(1.4-2.0)$ and 2.6 (2.0-3.0) for fathers in SSC and SC, respectively. Comparing fathers in the SC group by using paired statistics revealed a significant increase in the sub-scale spouse relationship 
problems from one to four months $(\mathrm{p}<0.05)$, medians (q1-q3) were $1.8(1.5-2.5)$ and $2.6(2.0-$ 3.0), respectively.

\section{Parental depression}

At four months corrected age, no significant differences were found between the groups in EPDS, neither between mothers nor between fathers (Table 4). The proportion of mothers with depressive symptoms (EPDS >11) was $6.3 \%$ in the SSC group and $6.7 \%$ in the SC group. Of fathers, 7.1\% in SSC and 8.3\% in SC had depressive symptoms (EPDS >9).

\section{Breastfeeding}

All mothers except one (SC group), stated that they during pregnancy had thought about breastfeeding and that their wish was to breastfeed. The feeding tube was revealed at a mean postmenstrual age of 258 (SD 9.7) days (36 weeks plus six days) in the SSC group and 259 (SD 8.3) days in the SC group (37 weeks). All infants ( $\mathrm{n}=18)$ in the SSC group were breastfeeding partly or exclusively at discharge, compared to 16 out of $19(84.2 \%)$ in the SC group (Table 5). More infants were breastfeeding partly or exclusively in the SSC group, compared to the SC group at one and four months, but the differences did not reach significance (Table 5).

\section{Discussion}

The present study is the first to report salivary cortisol results from preterm infants and their mothers when the infant has experienced SSC for an average of 19 hours per day during the first week of life, starting immediately after birth. One strength with the study was that parents consented to participate before randomisation to SSC or SC, which eliminated the risk of self-selection. 
Salivary cortisol reactivity was significantly lower in the SSC group compared to the SC group. The results indicate that almost continuous SSC including close parental contact and human touch, has a buffering effect on the infant's stress reactivity during handling at one month corrected age. This is in congruence with the theory that early physical contact has an impact on the infant's brain systems that manage stress $[8,22,28]$. This is also supported by a recent study from Feldman and co-workers, which shows that skin-to-skin contact for one hour a day during the first 14 days of a preterm infant's life has sustained effects with lower stress reactivity ten years later [27].

There was no difference in salivary cortisol reactivity between groups, in response to stillface. While the mothers may have had a buffering effect on the infants' stress reactivity during the nappy change, the still-face procedure includes a contrast situation because the mother is suddenly unavailable and not allowed to respond neither by facial expressions nor touch, and hence not accessible to buffer potential stress. Existing studies on still-face and cortisol reactivity also show various results including both cortisol responders and nonresponders $[8,62-65]$. Lewis and Ramsay found no significant cortisol reactivity in response to still-face [64]. Haley and Stansbury [65] found a significant increase when using a modified still-face procedure containing an additional still-face period, while Feldman et al found that the allowance of maternal touch during still-face decreased cortisol levels [8]. The still-face procedure is a situation considered to provoke negative emotions such as anger and frustration [42]. It has previously been suggested that sadness but not anger elicits an increase in cortisol levels [64], which leads to the need for further studies.

There was a positive correlation in cortisol levels between preterm infants and their mothers in SSC but not in SC, indicating a faster concordance of regularity in the SSC dyad. A concordance in salivary cortisol levels of preterm infants and their mothers has previously been reported, following an intervention involving co-care of the mother and infant around 
the clock [17]. It is plausible that the human touch and constant closeness to the parent day and night facilitate the preterm infant's biological brain development and the HPA axis maturation. The constant closeness may influence the regularity of a day-night rhythm between the parent and infant, and regulate cortisol levels accordingly. More infants in the SSC group were breastfed, but an impact of transferred cortisol through breast milk on the cortisol correlation between mother and infant has previously been elucidated $[14,15]$.

Even if the SC parents in the present study were also invited to stay overnight at the NICU and practice as much skin-to-skin contact as they wished, they could still choose how much, how often, and time of the day. When the parents choose, skin-to-skin contact may be practiced at time points that are primarily suitable for the parents (i.e. during daytime). This could potentially result in situations where the parents are not available for several hours, which may delay the dyad in tuning in to the same day-night rhythm.

We found no significant difference in total SPSQ or EPDS between groups. The most possible explanation for the non-significant result may be that although the birth of a preterm infant is a stressful event for parents, most parents may have recovered well by the time the infant has reached the corrected age of one or four months. Inclusion of parents of extremely preterm infants may have provided different results because extremely preterm infants have more medical problems, need more care and stay longer in the NICU. It has previously been suggested that fathers of preterm infants are at risk of developing stress-related symptoms when the infants are four months old [34], but we found no significant difference in total SPSQ or EPDS between groups. However, even if the SPSQ scores were rather low, fathers in the SC group scored significantly higher on the SPSQ subscale spouse relationship problems compared to fathers in the SSC group at four months. Moreover, they increased their score between the follow-ups at one and four months. In order to succeed with almost continuous 
SSC and make it practically feasible, both parents need to be involved in the care of the infant and alter the skin-to-skin contact. The parents are given a responsibility and they have to work together to make it work. Maybe this co-operation and the shared experience that is part of almost continuous SSC enhance a mutual understanding and support for each other's responsibilities, make the father more involved in the care, and strengthen the relationship between the parents. According to a study by Tessier et al., mothers are more likely to create a stimulating and better caregiving environment if they practice SSC. Further, more paternal support and involvement in the infants' care have a positive impact on the family context; families create a more stimulating environment and are more open to extended family and neighbours [29].

More infants in the SSC group were breastfed at discharge, at one month and four months, but the results did not reach significance. Positive effects of skin-to-skin contact on breastfeeding have been reported earlier but most studies are performed on full-term infants [66]. However, in a RCT study by Hake-Brooks and Anderson, low-birth weight infants with skin-to-skin contact were breastfed significantly longer and more exclusively compared to controls [30]. The infants in the study by Hake-Brooks and Anderson experienced skin-to-skin contact at a mean of 4.47 hours per day which is less than the infants in our control group. Thus, a plausible explanation for the non-significant results between SSC and SC is the many ours of skin-to-skin contact that the infants in our control group experienced. It should also be noted that the breastfeeding rates in our sample are rather high in both groups $[67,68]$. In a study by Carfoot et al., $43 \%$ of the infants who had experienced skin-to-skin contact and $40 \%$ in the control group were breastfed partly or exclusively at four months [67]. In the present study, the comparable figures were $76.5 \%$ and $53.3 \%$ for SSC and SC, respectively. 
Our study had some limitations. It was not possible to include a control group that did not practice skin-to-skin contact because allowing parents to practice as much skin-to-skin contact as as they like during their hospital stay is routine. This resulted in an average of 7.0 hours of skin-to-skin contact per day in the SC group, which is a lot compared to other studies, but significantly less than for the families randomised to SSC. One reason for the high use of skin-to-skin contact in the SC group could be the participation in the study itself. The study participants were all informed about the study and skin-to-skin contact before randomisation; a small intervention that possibly made the parents aware of the method and hence increased the use of skin-to-skin contact. Saliva was not collected from fathers because they generally are not the primary caregivers when the infants are four months old. However, since they also practice skin-to-skin contact, it would be beneficial to include them more in future studies, especially in order to investigate the salivary cortisol concordance between the infant and the father, compared to the concordance between the infant and the mother.

\section{Conclusions}

Early life factors, such as almost continuous SSC, can affect the development of the HPA axis. In the present study, almost continuous SSC decreased the salivary cortisol reactivity during a nappy change and improved the concordance between mothers' and infants' salivary cortisol levels, which could be a sign of a more rapid development of regularity. An intervention, such as almost continuous SSC, that involves and engages both parents immediately after the birth of a preterm infant and continues until discharge from the hospital seems to facilitates the spouse relationship.

\section{Acknowledgements}


The authors gratefully acknowledge the participating families, Lisbet de Jounge, Birgitta Lundin, Elisabeth Olhager, Ihsan Sarman, and staff members at the Neonatal Intensive Care Units at Linköping University Hospital and at Sachs' Children's Hospital in Stockholm. This study was supported by the County Council of Östergötland, South Sweden Nursing Society (SSSH), Hälsofonden, and Linköping University.

\section{Conflict of interest statement}

There are no conflicts of interest related to this paper. 


\section{References}

[1]Stevens BJ, Abbott LK, Yamada J, Harrison D, Stinson J, Taddio A, et al. Epidemiology and management of painful procedures in children in Canadian hospitals. CMAJ 2011;183(7):E403-10. [2] Lindberg B, Öhrling K. Experiences of having a prematurely born infant from the perspective of mothers in northern Sweden. Int J Circumpolar Health 2008;67(5):461-71.

[3] Christensson K, Cabrera T, Christensson E, Uvnas-Moberg K, Winberg J. Separation distress call in the human neonate in the absence of maternal body contact. Acta Paediatr 1995;84(5):468-73.

[4] Levine S. Developmental determinants of sensitivity and resistance to stress.

Psychoneuroendocrinology 2005;30(10):939-46.

[5] Lagercrantz H, Ringstedt T. Organization of the neuronal circuits in the central nervous system during development. Acta Paediatr 2001;90(7):707-15.

[6] Grunau RE, Haley DW, Whitfield MF, Weinberg J, Yu W, Thiessen P. Altered basal cortisol levels at 3, 6, 8 and 18 months in infants born at extremely low gestational age. The Journal of pediatrics 2007;150(2):151-6.

[7] Buss C, Entringer S, Swanson JM, Wadhwa PD. The Role of Stress in Brain Development: The Gestational Environment's Long-Term Effects on the Brain. Cerebrum : the Dana forum on brain science 2012;2012:4.

[8] Feldman R, Singer M, Zagoory O. Touch attenuates infants' physiological reactivity to stress.

Developmental science 2010;13(2):271-8.

[9] Liu D, Diorio J, Tannenbaum B, Caldji C, Francis D, Freedman A, et al. Maternal care, hippocampal glucocorticoid receptors, and hypothalamic-pituitary-adrenal responses to stress. Science 1997;277(5332):1659-62.

[10] Ros-Simo C, Valverde O. Early-life social experiences in mice affect emotional behaviour and hypothalamic-pituitary-adrenal axis function. Pharmacology, biochemistry, and behavior 2012;102(3):434-41.

[11] Neu M, Laudenslager ML, Robinson J. Coregulation in salivary cortisol during maternal holding of premature infants. Biol Res Nurs 2009;10(3):226-40.

[12] Thompson LA, Trevathan WR. Cortisol reactivity, maternal sensitivity, and learning in 3-monthold infants. Infant Behav Dev 2008;31(1):92-106.

[13] Yehuda R, Engel SM, Brand SR, Seckl J, Marcus SM, Berkowitz GS. Transgenerational effects of posttraumatic stress disorder in babies of mothers exposed to the World Trade Center attacks during pregnancy. J Clin Endocrinol Metab. 2005;90(7):4115-8.

[14] Spangler G. The emergence of adrenocortical circadian function in newborns and infants and its relationship to sleep, feeding and maternal adrenocortical activity. Early Hum Dev 1991;25(3):197208. 
[15] Stenius F, Theorell T, Lilja G, Scheynius A, Alm J, Lindblad F. Comparisons between salivary cortisol levels in six-months-olds and their parents. Psychoneuroendocrinology 2008;33(3):352-9. [16] Mörelius E, Theodorsson E, Nelson N. Stress at three-month immunization: parents' and infants' salivary cortisol response in relation to the use of pacifier and oral glucose. Eur J Pain 2009;13(2):2028.

[17] Mörelius E, Brostrom EB, Westrup B, Sarman I, Ortenstrand A. The Stockholm Neonatal Family-Centered Care Study: effects on salivary cortisol in infants and their mothers. Early Hum Dev 2012;88(7):575-81.

[18] Schreiber JE, Shirtcliff E, Van Hulle C, Lemery-Chalfant K, Klein MH, Kalin NH, et al. Environmental influences on family similarity in afternoon cortisol levels: twin and parent-offspring designs. Psychoneuroendocrinology 2006;31(9):1131-7.

[19] Custodio RJ, Junior CE, Milani SL, Simoes AL, de Castro M, Moreira AC. The emergence of the cortisol circadian rhythm in monozygotic and dizygotic twin infants: the twin-pair synchrony. Clin Endocrinol (Oxf) 2007;66(2):192-7.

[20] Örtenstrand A, Westrup B, Brostrom EB, Sarman I, Akerstrom S, Brune T, et al. The Stockholm Neonatal Family Centered Care Study: effects on length of stay and infant morbidity. Pediatrics 2010;125(2):e278-85.

[21] WHO. Kangaroo Mother Care: A Practical Guide. Geneva 2003.

[22] Feldman R, Weller A, Sirota L, Eidelman AI. Skin-to-Skin contact (Kangaroo care) promotes self-regulation in premature infants: sleep-wake cyclicity, arousal modulation, and sustained exploration. Dev Psychol 2002;38(2):194-207.

[23] Ludington-Hoe SM, Swinth JY. Developmental aspects of kangaroo care. J Obstet Gynecol Neonatal Nurs 1996;25(8):691-703.

[24] Mörelius E, Theodorsson E, Nelson N. Salivary cortisol and mood and pain profiles during skinto-skin care for an unselected group of mothers and infants in neonatal intensive care. Pediatrics 2005;116(5):1105-13.

[25] Neu M, Robinson J. Maternal holding of preterm infants during the early weeks after birth and dyad interaction at six months. J Obstet Gynecol Neonatal Nurs 2010;39(4):401-14.

[26] Johnston CC, Stevens B, Pinelli J, Gibbins S, Filion F, Jack A, et al. Kangaroo care is effective in diminishing pain response in preterm neonates. Archives of pediatrics \& adolescent medicine 2003;157(11):1084-8.

[27] Feldman R, Rosenthal Z, Eidelman AI. Maternal-Preterm Skin-to-Skin Contact Enhances Child Physiologic Organization and Cognitive Control Across the First 10 Years of Life. Biol Psychiatr 2013.

[28] Feldman R, Eidelman AI, Sirota L, Weller A. Comparison of skin-to-skin (kangaroo) and traditional care: parenting outcomes and preterm infant development. Pediatrics 2002;110(1 Pt 1):1626. 
[29] Tessier R, Cristo M, Velez S, Giron M, de Calume ZF, Ruiz-Palaez JG, et al. Kangaroo mother care and the bonding hypothesis. Pediatrics 1998;102(2):e17.

[30] Hake-Brooks SJ, Anderson GC. Kangaroo care and breastfeeding of mother-preterm infant dyads 0-18 months: a randomized, controlled trial. Neonatal Netw 2008;27(3):151-9.

[31] de Alencar AE, Arraes LC, de Albuquerque EC, Alves JG. Effect of kangaroo mother care on postpartum depression. Tropical Pediatrics 2009;55(1):36-8.

[32] Mörelius E, Angelhoff C, Eriksson J, Olhager E. Time of initiation of skin-to-skin contact in extremely preterm infants in Sweden. Acta Paediatr 2012;101(1):14-8.

[33] Nyqvist KH, Expert Group of the International Network on Kangaroo Mother C, Anderson GC, Bergman N, Cattaneo A, Charpak N, et al. State of the art and recommendations. Kangaroo mother care: application in a high-tech environment. Acta Paediatr 2010;99(6):812-9.

[34] Shaw RJ, Bernard RS, Deblois T, Ikuta LM, Ginzburg K, Koopman C. The relationship between acute stress disorder and posttraumatic stress disorder in the neonatal intensive care unit.

Psychosomatics 2009;50(2):131-7.

[35] Lasiuk GC, Comeau T, Newburn-Cook C. Unexpected: an interpretive description of parental traumas' associated with preterm birth. BMC pregnancy and childbirth 2013;13 Suppl 1:S13.

[36] Gray PH, Edwards DM, O'Callaghan MJ, Cuskelly M, Gibbons K. Parenting stress in mothers of very preterm infants -- influence of development, temperament and maternal depression. Early Hum Dev 2013;89(9):625-9.

[37] Bigelow A, Power M, MacLellan-Peters J, Alex M, McDonald C. Effect of mother/infant skin-toskin contact on postpartum depressive symptoms and maternal physiological stress. J Obstet Gynecol Neonatal Nurs 2012;41(3):369-82.

[38] Pearson RM, Melotti R, Heron J, Joinson C, Stein A, Ramchandani PG, et al. Disruption to the development of maternal responsiveness? The impact of prenatal depression on mother-infant interactions. Infant behavior \& development 2012;35(4):613-26.

[39] Weinberg MK, Beeghly M, Olson KL, Tronick E. Effects of Maternal Depression and Panic Disorder on Mother-Infant Interactive Behavior in the Face-to-Face Still-Face Paradigm. Infant Mental Health Journal 2008;29(5):472-91.

[40] Lovejoy MC, Graczyk PA, O'Hare E, Neuman G. Maternal depression and parenting behavior: a meta-analytic review. Clin Psychol Rev 2000;20(5):561-92.

[41] Neu M, Hazel NA, Robinson J, Schmiege SJ, Laudenslager M. Effect of holding on co-regulation in preterm infants: a randomized controlled trial. Early Hum Dev 2014;90(3):141-7.

[42] Gunnar MR, Talge NM, Herrera A. Stressor paradigms in developmental studies: what does and does not work to produce mean increases in salivary cortisol. Psychoneuroendocrinology 2009;34(7):953-67.

[43] Mörelius E, Nelson N, Gustafsson PA. Salivary cortisol response in mother-infant dyads at high psychosocial risk. Child: care, health and development 2007;33(2):128-36. 
[44] Tronick E, Als H, Adamson L, Wise S, Brazelton TB. The infant's response to entrapment between contradictory messages in face-to-face interaction. J Am Acad Child Psychiatry 1978;17(1):1-13.

[45] Ainsworth MB, SM; Stayton, D. Infant-mother attachment and social development. In: Richards M, editor. The introduction of the child into a social world London: Cambridge University Press; 1974. p. 99-135.

[46] Gunnar M, Brodersen L, Nachmias M, Buss K, Rigatuso J. Stress reactivity and attachment security. Dev Psychobiol 1996;29(3):191-204.

[47] Spangler G, Schieche M, Ilg U, Maier U, Ackermann C. Maternal sensitivity as an external organizer for biobehavioral regulation in infancy. Dev Psychobiol 1994;27(7):425-37.

[48] Albers EM, Riksen-Walraven JM, Sweep FC, de Weerth C. Maternal behavior predicts infant cortisol recovery from a mild everyday stressor. J Child Psychol Psychiatry 2008;49(1):97-103.

[49] Tu MT, Grunau RE, Petrie-Thomas J, Haley DW, Weinberg J, Whitfield MF. Maternal stress and behavior modulate relationships between neonatal stress, attention, and basal cortisol at 8 months in preterm infants. Dev Psychobiol 2007;49(2):150-64.

[50] Walker CD, Xu Z, Rochford J, Johnston CC. Naturally occurring variations in maternal care modulate the effects of repeated neonatal pain on behavioral sensitivity to thermal pain in the adult offspring. Pain 2008;140(1):167-76.

[51] Blomqvist YT, Rubertsson C, Nyqvist KH. Parent-infant skin-to-skin contact; How do parent records compare to nurse records? Acta Paediatr 2011;100(5):773-5.

[52] Mitchell EA. Co-sleeping and sudden infant death syndrome. Lancet 1996;348(9040):1466.

[53] Mörelius E, Hellstrom-Westas L, Carlen C, Norman E, Nelson N. Is a nappy change stressful to neonates? Early Hum Dev 2006;82(10):669-76.

[54] Tronick EZ, Messinger DS, Weinberg MK, Lester BM, Lagasse L, Seifer R, et al. Cocaine exposure is associated with subtle compromises of infants' and mothers' social-emotional behavior and dyadic features of their interaction in the face-to-face still-face paradigm. Dev Psychol 2005;41(5):711-22.

[55] Mörelius E, Nelson N, Theodorsson E. Salivary cortisol and administration of concentrated oral glucose in newborn infants: improved detection limit and smaller sample volumes without glucose interference. Scand J Clin Lab Invest 2004;64(2):113-8.

[56] Mörelius E, Nelson N, Theodorsson E. Saliva collection using cotton buds with wooden sticks: a note of caution. Scand J Clin Lab Invest 2006;66(1):15-8.

[57] Abidin RR. Parenting Stress Index. Charlottesville, VA: Pediatric Psychology Press; 1990.

[58] Östberg M, Hagekull B, Wettergren S. A measure of parental stress in mothers with small children: dimensionality, stability and validity. Scand J Psychol 1997;38(3):199-208. 
[59] Cox JL, Holden JM, Sagovsky R. Detection of postnatal depression. Development of the 10-item Edinburgh Postnatal Depression Scale. The British journal of psychiatry : the journal of mental science 1987;150:782-6.

[60] Wickberg B, Hwang CP. The Edinburgh Postnatal Depression Scale: validation on a Swedish community sample. Acta Psychiatrica Scandinavica 1996;94(3):181-4.

[61] Matthey S, Barnett B, Kavanagh DJ, Howie P. Validation of the Edinburgh Postnatal Depression Scale for men, and comparison of item endorsement with their partners. JAffective Disorders 2001;64(2-3):175-84.

[62] Tollenaar MS, Beijers R, Jansen J, Riksen-Walraven JM, de Weerth C. Maternal prenatal stress and cortisol reactivity to stressors in human infants. Stress 2011;14(1):53-65.

[63] Grant KA, McMahon C, Austin MP, Reilly N, Leader L, Ali S. Maternal prenatal anxiety, postnatal caregiving and infants' cortisol responses to the still-face procedure. Dev psychobiol 2009;51(8):625-37.

[64] Lewis M, Ramsay D. Infant emotional and cortisol responses to goal blockage. Child Dev 2005;76(2):518-30.

[65] Haley DW, Stansbury K. Infant stress and parent responsiveness: regulation of physiology and behavior during still-face and reunion. Child Dev. 2003;74(5):1534-46.

[66] Moore ER, Anderson GC, Bergman N, Dowswell T. Early skin-to-skin contact for mothers and their healthy newborn infants. The Cochrane database of systematic reviews. 2012;5:CD003519.

[67] Carfoot S, Williamson P, Dickson R. A randomised controlled trial in the north of England examining the effects of skin-to-skin care on breast feeding. Midwifery 2005;21(1):71-9.

[68] Cattaneo A, Burmaz T, Arendt M, Nilsson I, Mikiel-Kostyra K, Kondrate I, et al. Protection, promotion and support of breast-feeding in Europe: progress from 2002 to 2007. Public Health Nutrition 2010;13(6):751-9. 
Figures and tables

Figure 1

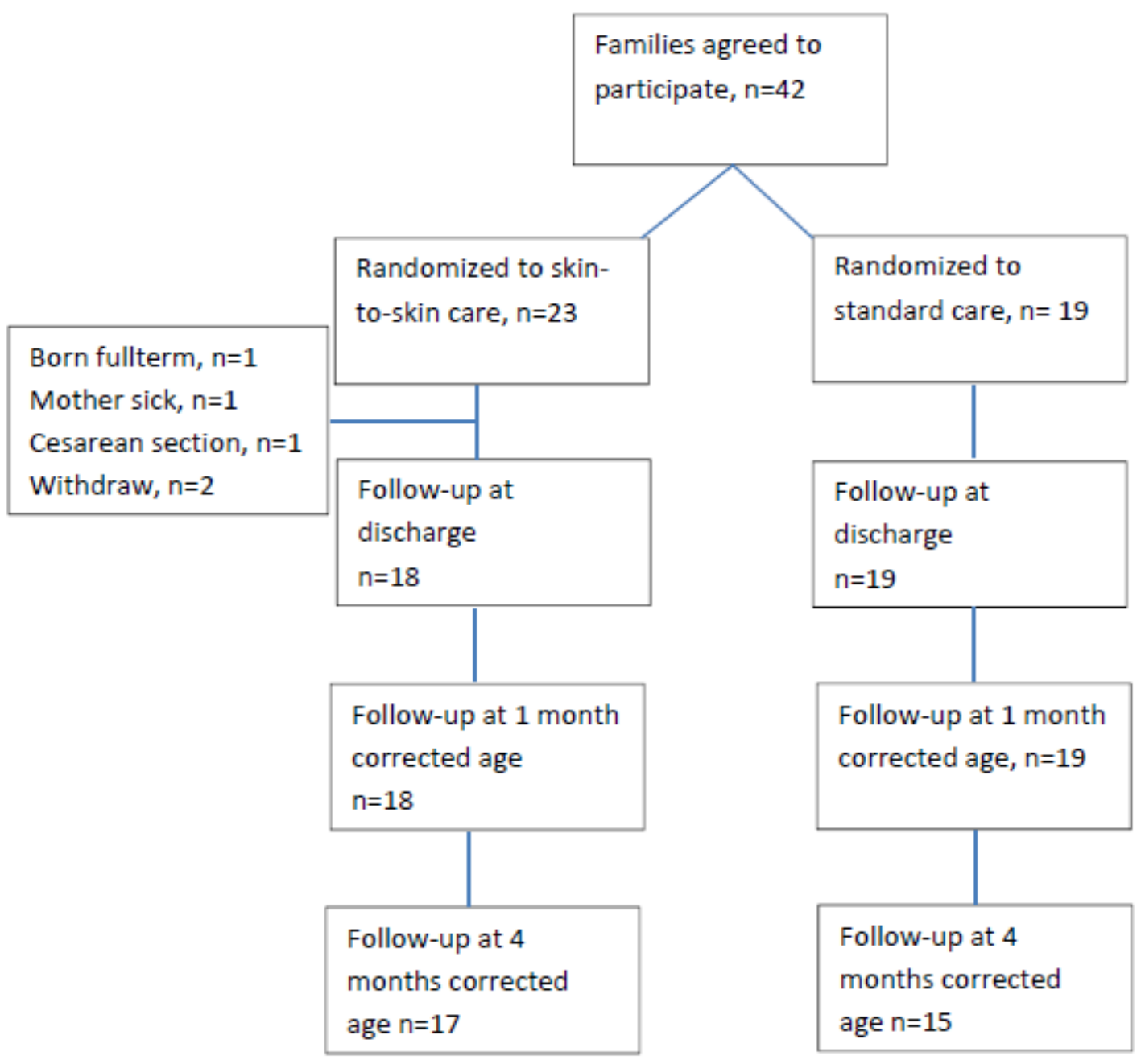

Figure 1: Flow chart 


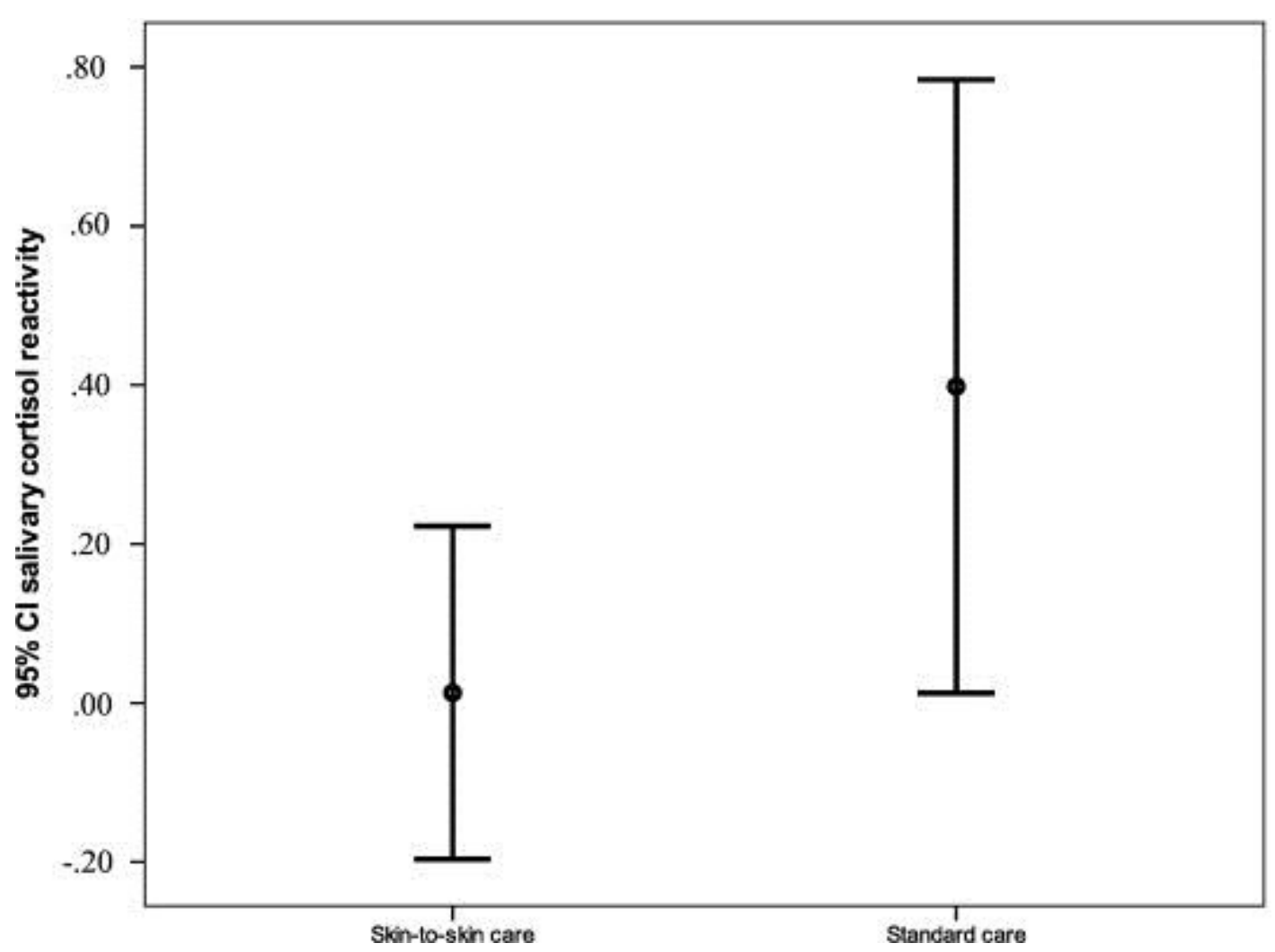

Figure 2: Error bars of salivary cortisol reactivity in response to nappy change at one month corrected age, for infants in SSC and SC group, respectively 
Table 1

\begin{tabular}{|c|c|c|c|}
\hline & $\begin{array}{l}\text { Skin-to-skin contact } \\
\mathrm{n}=18\end{array}$ & $\begin{array}{l}\text { Standard care } \\
\mathrm{n}=19\end{array}$ & \\
\hline Gender boys/girls & $11 / 7$ & $6 / 13$ & n.s. \\
\hline PMA at birth, mean (SD)/weeks & $243(6.2) / 34$ & $243(4.8) / 34$ & n.s. \\
\hline PMA at discharge, mean (SD)/weeks & $263(7.6) / 37$ & $262(4.8) / 37$ & n.s. \\
\hline Birth weight, g, mean (SD) & $2468(285)$ & $2512(274)$ & n.s. \\
\hline Weight at discharge, $g$, mean (SD) & $2811(336)$ & $2787(255)$ & n.s. \\
\hline Weight one month, g, mean (SD) & $4660(559)$ & 4649 (662) & n.s. \\
\hline Weight four months, $g$, mean (SD) & $6930(655)$ & $6715(860)$ & n.s. \\
\hline Siblings, yes/no/missing data & $5 / 13 / 0$ & $6 / 12 / 1$ & n.s. \\
\hline Mothers' age, yrs, mean (SD) & $31(3.5)$ & $33(5.3)$ & n.s. \\
\hline Fathers' age, yrs, mean (SD) & $33.5(5.2)$ & $34(5.1)$ & n.s. \\
\hline Cohabitate/single mother/missing data & $18 / 0 / 0$ & $17 / 1 / 1$ & n.s. \\
\hline \multicolumn{4}{|l|}{ Mothers' education } \\
\hline High school/university/other & $3 / 15 / 0$ & $3 / 15 / 1$ & n.s. \\
\hline \multicolumn{4}{|l|}{ Fathers' education } \\
\hline High school/university/other & $5 / 12 / 1$ & $6 / 11 / 2$ & n.s. \\
\hline \multicolumn{4}{|l|}{ Smoking, yes/no/missing data } \\
\hline Mothers & $0 / 18 / 0$ & $0 / 18 / 1$ & n.s. \\
\hline Fathers & $1 / 17 / 0$ & $1 / 16 / 2$ & n.s. \\
\hline
\end{tabular}

Table 1: Demographic and medical information of participating infants and mothers 
Table 2

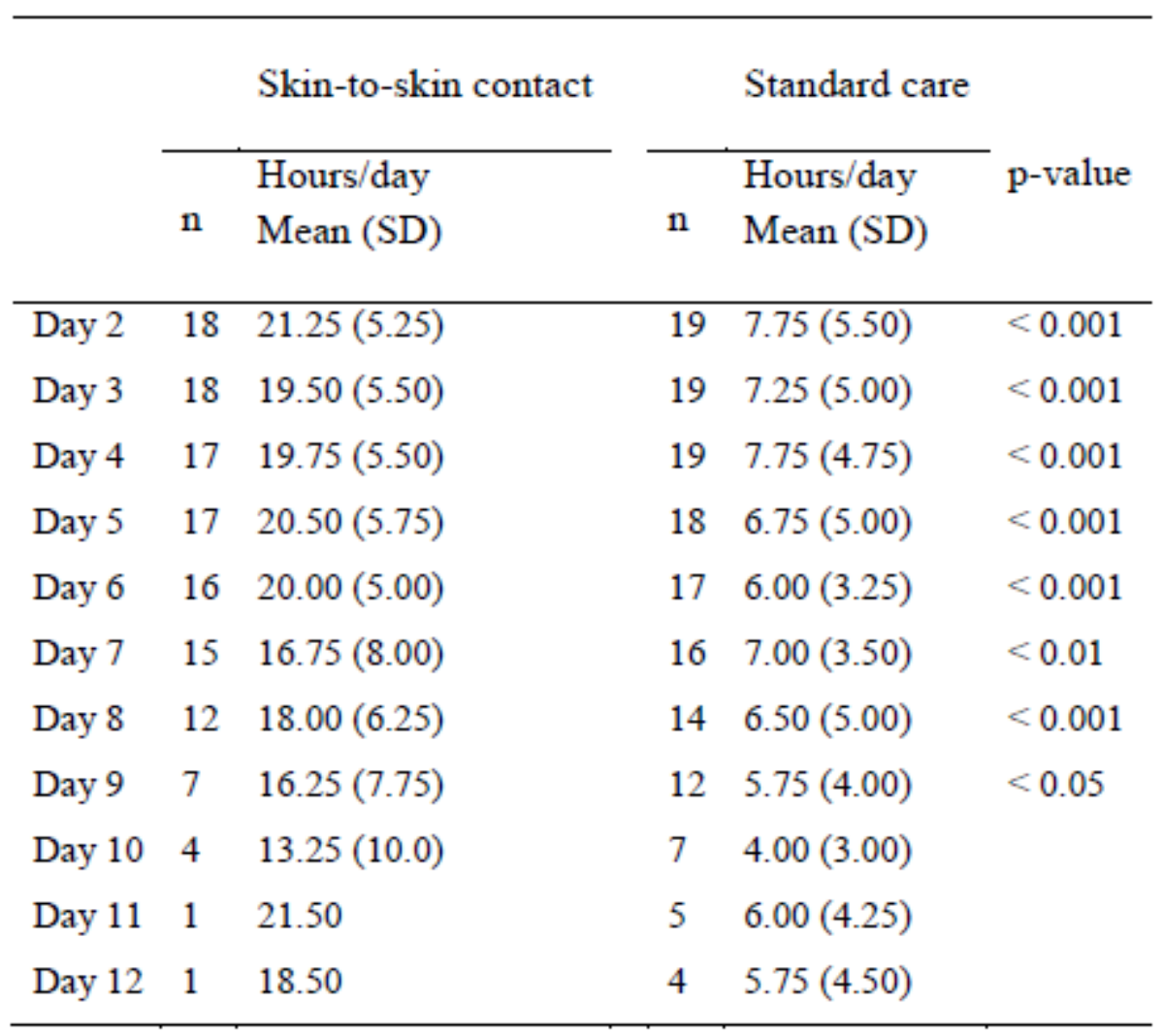

Table 2: Mean hours (SD) spent in skin-to-skin contact per day for the SSC group and SC group, respectively 
Table 3

\begin{tabular}{|c|c|c|c|c|c|c|}
\hline & \multicolumn{2}{|c|}{ Skin-to-skin contact } & \multicolumn{4}{|c|}{ Standard care } \\
\hline & Baseline & Response & Cortisol reactivity & Baseline & Response & Cortisol reactivity \\
\hline & Median (q1-q3) & Median (q1-q3) & & Median (q1-q3) & Median (q1-q3) & \\
\hline Infants 1 month & $6.1(5.0-16.8)$ & $7.3(4.3-11.7)$ & & $4.5(3.6-9.6)$ & $7.0(4.1-15.0)$ & \\
\hline Infants 4 months & $6.7(4.0-13.7)$ & $7.3(5.2-14.2)$ & & $7.5(5.3-15.5)^{\circ}$ & $6.8(4.3-16.6)$ & \\
\hline Mothers 4 months & $7.3(5.9-10.8)$ & $5.4(4.3-8.7)$ & & $6.7(5.6-8.3)$ & $5.4(4.2-6.1)$ & \\
\hline
\end{tabular}

- Significant increase from baseline at 1 month

Table 3: Infants' and mothers' raw salivary cortisol values

Table 4

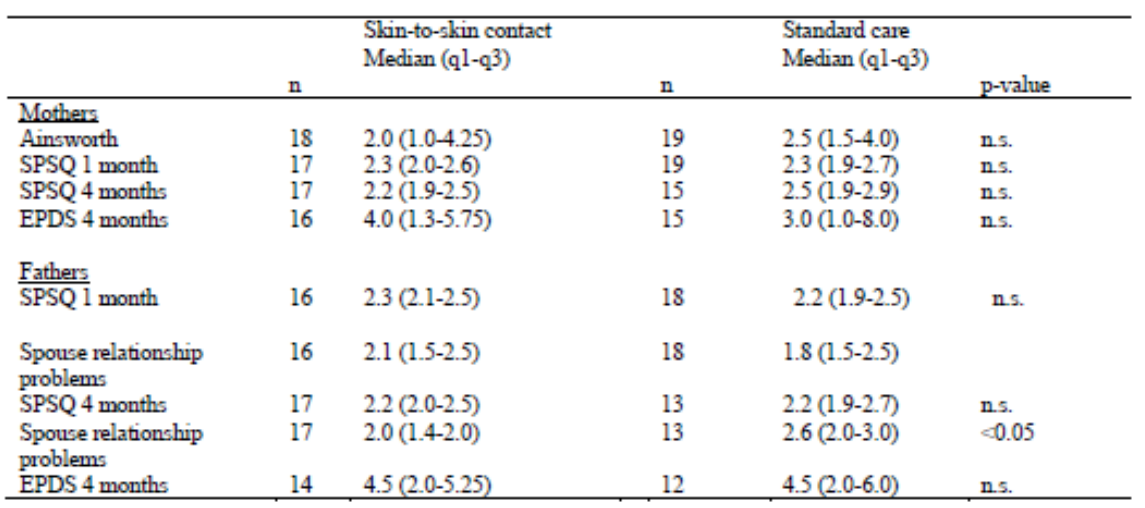

Table 4: Mothers' and fathers' parenthood stress (SPSQ) and depression (EPDS) scores at the follow-ups at one and four months 
Table 5

\begin{tabular}{lcclll}
\hline & \multicolumn{2}{c}{ Skin-to-skin care } & \multicolumn{2}{l}{ Standard care } & p-value \\
\hline Discharge, yes/no & $18 / 0$ & $100 \%$ & $16 / 3$ & $84.2 \%$ & n.s. \\
1 month, yes/no & $17 / 1$ & $94.4 \%$ & $14 / 5$ & $73.7 \%$ & n.s. \\
4 months, yes/no & $13 / 4$ & $76.5 \%$ & $8 / 7$ & $53.3 \%$ & n.s. \\
\hline
\end{tabular}

Table 5: Breastfeeding rates at discharge, one month corrected age, and four months corrected age for the skin-to-skin contact group and standard care group, respectively 